\title{
4D Active Surfaces for Cardiac Analysis
}

\author{
Anthony Yezzi ${ }^{1}$ and Allen Tannenbaum ${ }^{2}$ \\ 1 Dept. of Electrical \& Computer Eng., Georgia Tech, Atlanta, GA 30332-0250 \\ 2 Depts. of Electrical \& Computer and Biomedical Eng., Georgia Tech, Atlanta, \\ GA 30332-0250; and Dept. of EE, Technion, Haifa, Israel
}

\begin{abstract}
In this note, we employ the geometric active contour models formulated in [51119] for edge detection and segmentation to temporal MR cardiac images. The method is based on defining feature-based metrics on a given image which leads to a snake paradigm in which the feature of interest may be as the steady state of a curvature driven gradient flow. The implementation of the flow is done without level sets. This allow us to segment $4 \mathrm{D}$ sets directly, i.e., not as a series of $2 \mathrm{D}$ slices or a temporal series of $3 \mathrm{D}$ volumes.
\end{abstract}

\section{Introduction}

The technique of active contours or snakes has been employed for a number of purposes in medical imaging. Snakes are based upon the utilization of deformable contours which conform to various object shapes and motions. They have been used for edge and curve detection, segmentation, shape modelling, and visual tracking. A key application has been in medical imaging, in particular, the segmentation of endocardial heart boundary as a prerequisite from which such vital information such as ejection-fraction ratio, heart output, and ventricular volume ratio can be computed. See [6]916] and the references therein.

Ideally, one would want to segment $4 \mathrm{D}$ cardiac imagery directly as a $4 \mathrm{D}$ data set; see 20] and the references therein. Because of problems in speed and accuracy, to the best of our knowledge such data sets have been segmented either as sequence of $2 \mathrm{D}$ slices or a times series of $3 \mathrm{D}$ volumes. In this note, because of conformal snake algorithms based on the [5,11,19], and certain discretizations motivated partially by the work of Brakke [3], we feel that we can present practical algorithms for segmenting $4 \mathrm{D} \mathrm{MR}$ cardiac data as a $4 \mathrm{D}$ data set. Geometric active contour models involving curve evolution equations for segmentation were earlier proposed in 4,13. Briefly, the 4D conformal method is based on considering the gradient direction in which the 3D boundary of a $4 \mathrm{D}$ dimensional volume is shrinking as fast as possible relative to a conformal Euclidean metric. Thus, we multiply the Euclidean 3D area by a function tailored to the features of interest to which we want to flow, and compute resulting gradient evolution equations. This will be explicated in the next section.

Besides the application to 4D segmentation, we believe that the methods in this paper may have independent interest for various segmentation tasks in medical imaging, especially those involving geometric partial differential equations defined as gradient flows from the minimization of energies based on geometric properties (length, area, volume, etc.). Indeed, all the gradient snake segmentations performed in this paper, were done without level sets. This is important

T. Dohi and R. Kikinis (Eds.): MICCAI 2002, LNCS 2488, pp. 667673 2002.

(C) Springer-Verlag Berlin Heidelberg 2002 
since we were able to perform our $4 \mathrm{D}$ segmentations very quickly: we tested 4 data sets each $256 \times 256 \times 18 \times 12$ (the last being the time parameter), with each segmentation taking about 25 seconds on a standard Sun Ultrasparc 60. More details will be given below.

Of course, we do not propose this methodology as a replacement for level sets [15], which have a number of attractive properties (including perhaps the best method for interface tracking), but rather as a complementary technique for certain specific problems such as that sketched in this paper.

We now briefly outline the contents of this paper. In Section 2, we describe the gradient (geodesic) active contour framework in $4 \mathrm{D}$. In Section 3, we then discuss our discrete model of this evolution equation, and then in Section 4 we apply it to $4 \mathrm{D}$ cardiac MR imagery. Finally, we draw some conclusions in Section 5 .

\section{4D Gradient Snakes}

In this section, we formally derive the $4 \mathrm{D}$ conformal active contour model based on a modified mean curvature flow.

Indeed, let $\phi: \Omega \rightarrow \mathbf{R}$ be a positive differentiable function defined on some open subset of $\mathbf{R}^{4}$. The function $\phi(x, y, z, w)$ will play the role of a "stopping" function, and so depends on the given grey-level image. Explicitly, the term $\phi(x, y, z, w)$ may chosen to be small near a $4 \mathrm{D}$ edge, and so acts to stop the evolution when the $3 \mathrm{D}$ contour reaches the edge. A standard choice would be

$$
\phi:=\frac{1}{1+\left\|\nabla G_{\sigma} * I\right\|^{2}},
$$

where $I=I(x, y, z, w)$ is the (grey-scale) volumetric image and $G_{\sigma}$ is a Gaussian (smoothing) filter.

The basic idea for $4 \mathrm{D}$ active contours is to replace the Euclidean surface area by a modified (conformal) area depending on $\phi$ namely,

$$
d S_{\phi}:=\phi d S .
$$

Indeed, for a family of surfaces (with parameter $t$ ), consider the $\phi$-area functional

$$
A_{\phi}(t):=\iint_{S} d S_{\phi} .
$$

Then taking the first variation and using a simple integration by parts argument, we get that gradient flow is given by

$$
\frac{\partial S}{\partial t}=(\phi H-\nabla \phi \cdot \mathcal{N}) \mathcal{N},
$$

where $H$ denotes the mean curvature (sum of the principle curvatures; see 8]) and $\mathcal{N}$ the unit normal. Notice that Euclidean conformal area $d S_{\phi}$ is small near an edge. Thus we would expect and initial $4 \mathrm{D}$ contour to flow to the potential well indicated by the evolution (2). As in [413], a constant inflation term $\nu$ may be added to give the model

$$
\frac{\partial S}{\partial t}=(\phi(H+\nu)-\nabla \phi \cdot \mathcal{N}) \mathcal{N} .
$$


This inflationary constant may be taken to be either positive (inward evolution) or negative in which case it would have an outward or expanding effect.

The flow from the inflationary constant in fact also can be derived as a gradient flow, this time one minimizing conformally weighted volume; see, e.g., [21].

\section{Discrete Implementation}

Level set methods introduced by Osher and Sethian [15] are powerful engines for evolving interfaces. In our case, one would have to evolve $4 \mathrm{D}$ surfaces in $\mathbf{R}^{5}$ in which the 3D active surfaces in which we are interested would be the zero level sets. There are methods based on taking narrow bands to handle such problems ([18]), and there is fast marching for the inflationary part of the flow 1822 which would make level sets more practical in our case. Because of noise in the image, we have found that one needs the curvature part throughout the evolution and so we cannot use fast marching, and the narrow band methods were difficult to implement because of the complicated $4 \mathrm{D}$ topology and the frequent need for re-initialization.

We therefore decided to use a discretization method based on ideas on Ken Brakke for directly minimizing the underlying energy functional, and using some elementary ideas in discrete geometry 3]. Essentially, we minimize the conformal functional using a conjugate gradient method.

In order to make our procedure fast and reliable we need corresponding algorithms to compute the mean curvature and normal vectors. For simplicity and for ease of visualization we explain the method in 3D. The extension to 4D (and even $n$-D) is immediate.

The basic geometric elements we use are vertices, edges, faces, and bodies. These elements are referred to by identifiers which also contain an orientation for each element. Controlling surface topology is essential in our method since we want to be able to include automatic merging and breaking of contours. When one starts from the growth of a single or several seeds in capturing a given structure, there may be a number of topological changes that must be automatically be built into the algorithm. In the methodology we employ, the surface topology is gotten by cross-references among the elements. In our case, each face-edge is in two double-linked list, one of face-edges around the edge, and another of face-edges around the face. Every edge points to its two endpoints, and to one face-edge loop around it. Each face points to one face-edge in the face-edge loop around it, and to the two bodies on its two sides. Each body points to one face-edge on its boundary. This allows us to keep track of the evolving surface and also to apply simple logical rules for splitting and merging. In doing this, we employ both global and local information.

At each point on an $n$-dimensional (hyper)surface, there are $n$ principal curvatures, the eigenvalues of the shape operator. The mean curvature is then given by their sum. The mean curvature vector $H \mathcal{N}$ has a very simple geometric characterization which makes it easy to compute. Indeed, as alluded to above it gives the direction in which the surface area is shrinking as fast as possible using only local information. Therefore, we can find the mean curvature vector on a 
triangulated surface by taking the direction in which the the area will be decreasing most rapidly, and then given the length of this vector, we can compute the normal direction.

This leads to the following procedure for computing mean curvature on a triangulated surface. Let $v$ be a vertex. Each vertex has a star of faces around it of area $A_{v}$. The force due to the surface tension on the vertex is given by

$$
F_{v}=-\frac{\partial A_{v}}{\partial v} .
$$

Since each face has three vertices, the area associated with $v$ is $A_{v} / 3$. Thus the (average) mean curvature at $v$ is

$$
h_{v}=\frac{3}{2} \frac{F_{v}}{A_{v}} .
$$

We also use the Gaussian curvature (the product of the principal curvatures). At a vertex of a triangulated surface, this is very easy to compute as

$$
\left(\sum_{i} \theta_{i}\right)-\pi
$$

where the sum is taken over the adjacent angles to a given vertex. This allows one to decide whether the surface is convex or concave at a given point (convex means that the about quantity will be $<\pi$, and concave $>\pi$ ). We should note that for hypersurfaces in 4D (the case of interest in our cardiac segmentation), we also compute the curvature defined by taking the sum of the products of the principal curvatures taken two at a time. In this case, these curvatures allow us to classify points as elliptic, hyperbolic, or parabolic.

Full details of our discrete implementation of the 4D conformal snakes will be reported in a forthcoming publication.

\section{Smoothing the Gradient}

For noisy images, some type of smoothing of the data may be necessary. This could be accomplished by using various types of anisotropic filters.

We also used a nice method reported in [25]. One can deal with the problem on which the $n \mathrm{D}$ snake may get caught by smoothing the gradient term $\nabla \phi$ in the following manner. (We work out the general case here. For the $4 \mathrm{D}$ cardiac, one can take $n=4$.) Let

$$
v\left(x_{1}, \ldots, x_{n}\right)=\left(v_{1}\left(x_{1}, \ldots, x_{n}\right), \ldots, v_{n}\left(x_{1}, \ldots, x_{n}\right)\right),
$$

be the desired smoothed vector field. Then $v\left(x_{1}, \ldots, x_{n}\right)$ is derived as the minimal energy solution of the following variational problem:

$$
\iint 2 \alpha \sum_{i=0}^{n}\left\|\nabla v_{i}\right\|+\|\nabla \phi\|^{2}\|v-\nabla \phi\|^{2} d x_{1} \ldots d x_{n} .
$$

This minimization problem may be solved using gradient descent associated Euler-Lagrange equations:

$$
\alpha \operatorname{div}\left(\frac{v_{i}}{\left\|v_{i}\right\|}\right)-\left(v_{i}-\nabla \phi\right)\|\nabla \phi\|^{2}=0, \quad i=1, \ldots, n .
$$


Note that for a function $f: \mathbf{R}^{n} \rightarrow \mathbf{R}$ we set

$$
\operatorname{div} f:=\sum_{i=0}^{n} \frac{\partial f}{\partial x_{i}} .
$$

Here $\alpha$ acts as a scale space parameter: the larger the $\alpha$ the more the smoothing effect on the gradient vector field.

\section{$5 \quad$ Endocardium Segmentation Results}

We now describe the results applied to temporal 3D cardiac imagery. A cardiac data set was acquired consisting of 12 time points and 18 spatial locations on a normal volunteer in a $1.5 \mathrm{~T}$ MR imaging system using a segmented $k$-space sequence. The short axis slices were $5 \mathrm{~mm}$ thick and the field of view was $32 \mathrm{~cm}$ with a data matrix of $256 \times 256$. A body coil was used to provide a uniform signal intensity. The left hand blocks of Figures 1 and 2 indicate original data slices. The vertical columns are the time series, and the horizontal indicate the change in volumetric spatial location. The corresponding endocardial contours are indicated in the right hand blocks of Figures 1 and 2.

Only one initial contour on a single $2 \mathrm{D}$ slice was necessary to initialize this process. The total time for the segmentation took about 25 seconds on an Ultrasparc 60 machine. Note that some portions of the detected contours (which are really cross sections of a single 4 dimensional active surface) appear thicker than in other places. These correspond to regions in which the $4 \mathrm{D}$ surface runs tangentially to the plane of the 2D images. We notice the benefit the full spatial and temporal coherency, that is automatically ensured by using a single 4D surface rather than multiple $2 \mathrm{D}$ curves, particularly well by observing the results in the lower left 2D image of the sequence near the base of the heart. In this image we can see the dark mitrovalve in the middle of the bright blood pool. Furthermore, the edge of the endocardial wall is very weak near the bottom of the image. However, the final contour is not confused by the dark mitrovalve and successfully detects even the weak portions of the endocardial boundary.

\section{Conclusions}

In this paper, we indicated that with fast geometric contour based methods one can derive an efficient procedure for the segmentation of the endocardium from MR 4D data, and thus a very quick way of computing the ejection fraction.

We employed the gradient snake model of [511 19] but did not use the standard level set implementation. Instead of evolving a four dimensional hypersurface in $\mathbf{R}^{5}$, we instead used a method that did not increase the dimensionality of the problem. Our methodology was strongly motivated by the direct energy minimization ideas as described in 3 .

For 4D MR endocardium segmentation, this approach seems to present an attractive alternative to the standard level set framework. Complete details about our algorithm will be presented in an upcoming publication. 

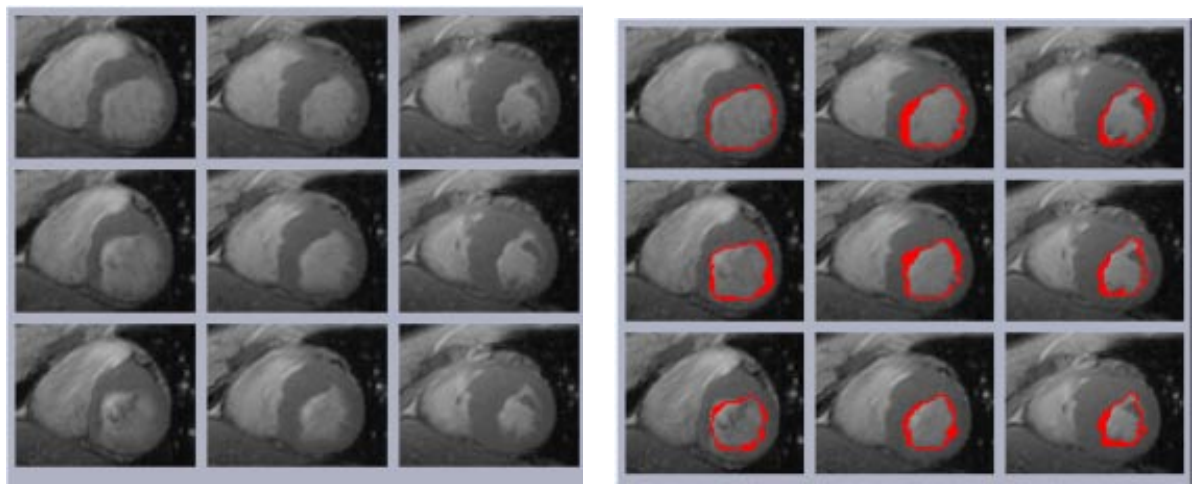

Fig. 1. Original Sequence Near Base of Heart (left) and Segmentation (right). This is a color image.
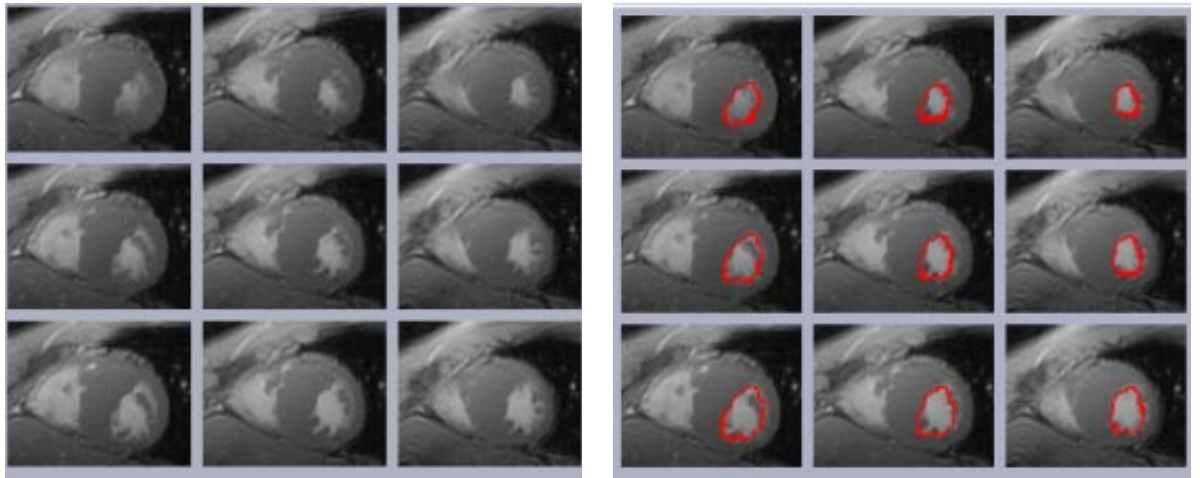

Fig. 2. Original Sequence Near Apex of Heart (left) and Segmentation (right). This is a color image.

\section{References}

1. A. Blake and A. Yuille, Active Vision, MIT Press, Cambridge, Mass., 1992.

2. K. Brakke, Motion of a Surface by its Mean Curvature, Princeton University Press, Princeton, NJ, 1978.

3. K. Brakke, Surface Evolver Manual, Research Report GCC 17, Geometry Center, University of MInnesota, 1990.

4. V. Caselles, F. Catte, T. Coll, and F. Dibos, "A geometric model for active contours in image processing," Numerische Mathematik 66, pp. 1-31, 1993.

5. Caselles, R. Kimmel, and G. Sapiro, , "Geodesic snakes," International Journal of Computer Vision, 1997.

6. H. Cline, R. Hartley, and R. Curwen, Fourth ISMRM Conference, p. 1623, 1996.

7. L. D. Cohen, "On active contour models and balloons," CVGIP: Image Understanding 53, pp. 211-218, 1991.

8. M. P. Do Carmo, Differential Geometry of Curves and Surfaces, Prentice-Hall, Inc., New Jersey, 1976. 
9. A. Gupta, L. von Kurowski, A. Singh, D. Geiger, C. Liang, M. Chiu, P. Adler, M. Haacke, and D. Wilson, "Cardiac MRI analysis: segmentation of myocardial boundaries using deformable models," Technical Report, Siemens Corporate Research, Princeton, NJ, 1995.

10. M. Kass, A. Witkin, and D. Terzopoulos, "Snakes: active contour models," 'Int. Journal of Computer Vision 1, pp. 321-331. 1987.

11. S. Kichenassamy, P. Olver, A. Tannenbaum, and A. Yezzi, "Conformal curvature flows: from phase transitions to active vision," Archive of Rational Mechanics and Analysis 134 pp. 275-301, 1996.

12. B. B. Kimia, A. Tannenbaum, and S. W. Zucker, "On the evolution of curves via a function of curvature, I: the classical case," J. of Math. Analysis and Applications 163, pp. 438-458, 1992.

13. R. Malladi, J. Sethian, and B. Vemuri, Shape modeling with front propagation: a level set approach, IEEE Trans. Pattern Anal. Machine Intell. 17, pp. 158-175, 1995.

14. S. Osher, "Riemann solvers, the entropy condition, and difference approximations," SIAM J. Numer. Anal. 21, pp. 217-235, 1984.

15. S. J. Osher and J. A. Sethian, "Fronts propagation with curvature dependent speed: Algorithms based on Hamilton-Jacobi formulations," Journal of Computational Physics 79, pp. 12-49, 1988.

16. S. Raganath, "Contour extraction from cardiac MRI studies using snakes," IEEE Trans. on Medical Imaging 14, pp. 328-338, 1995.

17. G. Sapiro and A. Tannenbaum, "On invariant curve evolution and image analysis," Indiana Univ. Journal of Math. 42, 1993, pp. 985-1009.

18. J. A. Sethian, Level Set Methods and Fast Marching Methods, Cambridge Press, Cambridge, 1999.

19. J. Shah, "Recovery of shapes by evolution of zero-crossings," Technical Report, Dept. of Mathematics, Northeastern University, Boston, MA, 1995.

20. P. Shi, G. Robinson, A. Chakraborty, L. Staib, R. T. Constable, A. Sinusas, and J. Duncan, "A unified framework to assess myocardial function from 4D Images," Lecture Notes in Computer Science: First International Conference on Computer Vision, Virtual Reality, and Robotics in Medicine (1995), pp. 327-337.

21. Y. Lauzier, K. Siddiqi, A. Tannenbaum, and S. Zucker, "Area and length minimizing flows for segmentation," IEEE Trans. Image Processing 7 (1998), pp. 433-444.

22. J. Tsitsiklis, "Efficient algorithms for globally optimal trajectories," IEEE Trans. Aut. Control 40 (1995), pp. 1528-1538.

23. H. Tek and B. Kimia, "Deformable bubbles in the reaction-diffusion space," Technical Report 138, LEMS, Brown University, 1995.

24. A. Yezzi, S. Kichenesamy, A. Kumar, P. Olver, and A. Tannenbaum, "Geometric active contours for segmentation of medical imagery," IEEE Trans. Medical Imaging 16 (1997), pp. 199-209.

25. C. Xu and J. Prince, "Snakes, Shapes, and Gradient Vector Flow," IEEE Trans. Image Processing 7 (1998), pp. 359-369. 